\title{
ON THE NUMBER OF FIELD TOPOLOGIES ON AN INFINITE FIELD
}

\author{
JOHN O. KILTINEN
}

Abstract. K.-P. Podewski has recently proven that every countable infinite field admits $2^{2 \boldsymbol{N}_{0}}$ different field topologies. Using methods of valuation theory, it is proven that every uncountable field, and more generally, every field $F$ of infinite transcendence degree over some subfield, admits $2^{2^{|F|}}$ field topologies. By purely set theoretic considerations, it then follows that there are $2^{2}|F|$ field topologies on any infinite field $F$, no two of which are topologically isomorphic. This latter result is then generalized to any infinite commutative ring without proper zero-divisors. A further aspect of Podewski's work on countable fields is generalized in a final theorem which states that a field $F$ of infinite transcendence degree admits $2^{2^{|P|}}$ field topologies which fail to be suprema of locally bounded ring topologies.

1. Introduction. This paper extends to the uncountable case some recent results of K.-P. Podewski [7] on the number of field topologies on a countable infinite field. One then has that any infinite field $F$ admits $2^{2}{ }^{|m|}$ field topologies, the maximum number possible.

Our methods of proof follow Podewski's in identifying a class of $2^{\left|\boldsymbol{F}^{\mid}\right|}$ topologies on $F$ which are interrelated so that the suprema of distinct subclasses are distinct. However, for uncountable fields, and more generally for fields of infinite transcendence degree over a subfield, one is able to use a class of $2^{|F|}$ valuation topologies, ${ }^{1}$ and the details of the proof become much easier than in the countable case. Valuation topologies are clearly not directly applicable to obtaining $2^{2^{101}}$ topologies which are not suprema of locally bounded ones, so to do this, we turn in $\S 3$ to the inductive ring topologies developed in [4], [5], and [6].

Presented to the Society, January 26, 1973 ; received by the editors November 16, 1972. AMS (MOS) subject classifications (1970). Primary 12J99, 13J99; Secondary 12J20, 12F20, 54A25, 54A10, 04A10.

Key words and phrases. Number of field topologies, valuation, inductive ring topology, locally bounded, infinite transcendence degree, supremum of a family of topologies, finite intersection property with complements.

${ }^{1}$ The author wishes to acknowledge the suggestion by the referee of an earlier version of this paper that valuation topologies can be used here. A proof previously based on the inductive topologies discussed in $\$ 3$ was thereby greatly simplified.

(c) American Mathematical Society 1973 
We state here two requisite known theorems-one from valuation theory and the other from set theory. We will consider valuations as mapping into the ordered multiplicative group of positive real numbers, with zero adjoined.

THEOREM 1.1. Let B be a transcendence basis over a subfield for a field $F$. Then for any function $f$ from $B$ into $\boldsymbol{R}_{+}^{*}$, the positive real numbers, there is a valuation $v_{f}$ on $F$ such that $v_{f}(x)=f(x)$ for every $x$ in $B$.

This theorem can be proven by transfinite induction using standard methods from valuation theory found in a reference such as [8].

Definition. Let $X$ be a set and let $\mathscr{S}$ be a subset of $\mathscr{P}(X)$, the power set of $X$. We will say that $\mathscr{S}$ has the finite intersection property with complements (f.i.p.c.) if for each pair of natural numbers $m$ and $n$ and each pairwise distinct collection of sets $S_{1}, S_{2}, \cdots, S_{m}, T_{1}, T_{2}, \cdots, T_{n}$ from $\mathscr{S}$, the set $\left(\bigcap_{i=1}^{m} S_{i}\right) \cap\left(\bigcap_{j=1}^{n} T_{j}^{c}\right)$ is not empty. Here $T_{j}^{c}$ denotes the complement of $T_{j}$ in $X$.

THEOREM 1.2 (HAUSDORFF [2]). If $X$ is an infinite set, then there exists a subset $\mathscr{S}$ of $\mathscr{P}(X)$ such that $\mathscr{S}$ has the f.i.p.c. and $|\mathscr{S}|=2^{|X|}$.

\section{The main results.}

THEOREM 2.1. Let $F$ be an infinite field of transcendence degree $|F|$ over a subfield $K$. Then there are $2^{2^{|F|}}$ distinct field topologies on $F$, each of which is the supremum of a family of valuation topologies.

Proof. Let $B$ be a transcendence basis for $F$ over $K$, and let $X$ be an index set such that $|X|=|B|$. Since $B$ is infinite, we may choose countably many subsets $B_{i}$ of $B$ such that $B=\bigcup_{i=1}^{\infty} B_{i}, B_{i} \cap B_{j}=\varnothing$ if $i \neq j$, and $\left|B_{i}\right|=|X|$ for each $i$. For each $i$, let $\phi_{i}: X \rightarrow B_{i}$ be a bijection. Let $\mathscr{S}$ be a subset of $\mathscr{P}(X)$ with the f.i.p.c. such that $|\mathscr{S}|=2^{|X|}$.

Now for each $S \in \mathscr{S}$, let $f_{S}: B \rightarrow R_{+}^{*}$ be defined by

$$
\begin{aligned}
f_{S}(x) & =1 / n & & \text { if } x \in \phi_{n}(S), \\
& =1 & & \text { otherwise. }
\end{aligned}
$$

Then by Theorem 1.1, there is a valuation $v_{S}$ on $F$ which agrees on $B$ with $f_{S}$. Let the topology on $F$ induced by $v_{S}$ be denoted by $\mathscr{T}_{S}$. Note that for any $\alpha$ in $X$, the sequence $\left(\phi_{n}(\alpha)\right)$ in $B$ converges to zero in $\mathscr{T}_{S}$ if $\alpha \in S$ and is bounded from zero otherwise.

We now let $\mathscr{T}_{\mathcal{O}}$ denote the supremum of the family $\left\{\mathscr{T}_{S} \mid S \in \mathcal{O}\right\}$ for each subset $\mathcal{O}$ of $\mathscr{S}$. We will show that each of these $2^{2 / 1 / 4}$ field topologies $\mathscr{T}_{\mathscr{O}}$ on $F$ is distinct.

To see this, suppose $\mathcal{O}_{i} \subseteq \mathscr{S}$ for $i=1,2$, and that $\mathcal{O}_{1} \neq \mathcal{O}_{2}$. Let us suppose 
in particular that there is a subset $S_{0}$ of $X$ such that $S_{0} \in \mathcal{O}_{1}$ and $S_{0} \notin \mathcal{O}_{2}$. It will follow that $\mathscr{T}_{\mathcal{O}_{1}} \neq \mathscr{T}_{\mathcal{C}_{2}}$, and hence $\mathscr{T}_{\mathcal{O}_{1}} \neq \mathscr{T}_{\mathcal{O}_{2}}$.

Suppose to the contrary that $\mathscr{T}_{\mathcal{O}_{1}} \subseteq \mathscr{T}_{\mathcal{O}_{2}}$. Let $U=\left\{x \in F \mid v_{S_{0}}(x)<\frac{1}{2}\right\}$. Then $U$ is a $\mathscr{T}_{S_{0}{ }^{-}}$, and hence also a $\mathscr{T}_{\mathscr{C}_{i}}$-neighborhood of zero, for $i=1$ and 2. Hence, there are sets $S_{1}, S_{2}, \cdots, S_{n}$ in $\mathcal{O}_{2}$ and $\varepsilon>0$ such that $\bigcap_{i=1}^{n} V_{i} \subseteq U$, where $V_{i}=\left\{x \in F \mid v_{S_{i}}(x)<\varepsilon\right\}$. Let $m$ be an integer such that $1 / m<\varepsilon$.

Now since $\mathscr{S}$ has the f.i.p.c., there is an element $\alpha$ in $\left(\bigcap_{i=1}^{n} S_{i}\right) \cap S_{0}^{c}$. For each $i, 1 \leqq i \leqq n, \phi_{m}(\alpha) \in \phi_{m}\left(S_{i}\right)$, so $v_{S_{i}}\left(\phi_{m}(\alpha)\right)=1 / m$. Thus,

$$
\phi_{m}(\alpha) \in \bigcap_{i=1}^{n} V_{i} \text {. }
$$

However, since $\alpha \notin S_{0}, \phi_{m}(\alpha) \notin \phi_{m}\left(S_{0}\right)$, so $v_{S_{0}}\left(\phi_{m}(\alpha)\right)=1$, and hence $\phi_{m}(\alpha) \notin U$. This is a contradiction, so we conclude that $\mathscr{T}_{\mathcal{O}_{1}} \ddagger \mathscr{T}_{\mathcal{O}_{2}}$.

Since an uncountable field $F$ satisfies the conditions of Theorem 2.1, taking $K$ to be the prime subfield, from Podewski's result and the above theorem, we have the following

COROLLARY 2.2. Every infinite field $F$ admits $2^{2^{|x|}}$ distinct field topologies.

We note that in the proof of Theorem 2.1, the topologies $\mathscr{T}_{\mathscr{O}}$ distinguished themselves from each other by their behavior on $B$, and thus, their restrictions to any subring of $F$ which contains $B$ will remain distinct. This suggests the following generalization.

THEOREM 2.3. If $R$ is any infinite commutative ring without proper zero-divisors, then there are $2^{2^{|R|}}$ distinct ring topologies on $R$ for which (if $R$ has an identity element) multiplicative inversion is continuous everywhere that it is defined.

Proof. Let $F$ denote a quotient field of $R$, let $K$ be the prime subfield of $F$. It is easily seen that there exists a transcendence basis $B$ for $F$ over $K$ such that $B \subseteq R$. If $B$ is infinite (in particular, if $R$ is uncountable) then as was observed above, the $2^{2^{|P|}}=2^{2^{|R|}}$ distinct topologies on $F$ presented in Theorem 2.1 have distinct restrictions to $R$. These relative topologies are, of course, ring topologies for which multiplicative inversion is continuous where it is defined.

The proof for the case where $R$ and $F$ are countable is based on the fact that the $2^{2^{\aleph_{0}}}$ topologies which Podewski produces on $F$ actually distinguish themselves from each other by their behavior on a certain inductively defined set. (The set is the union over all $n \in N$ and all $f \in{ }^{n} 2$ of $p^{f}(0,1) \cap\left(\bigcap\left\{p^{g}(n, 0) \mid g \in n^{2}\right.\right.$ and $\left.g \neq f\right\}$. See the development after Theorem 12 in [7].) At the $n$th stage in the definition of this set, one chooses finitely many elements, subject to certain restrictions keeping 
them out of certain finite subsets of $F$. Let us now impose the further restriction that these elements be chosen from $R$. It then follows that the $2^{2 N_{0}}$ distinct topologies on $F$ remain distinct when relativized to $R$.

We conclude this section with the observation that any infinite field $F$ admits $2^{2^{|f|}}$ field topologies which are really distinct from each other in the sense of not being topologically isomorphic. This follows from Corollary 2.2 via purely set-theoretic considerations.

THEOREM 2.4. If $F$ is an infinite field, then there is a set $\Sigma$ of $2^{2^{|m|}}$ field topologies on $F$ such that if $\mathscr{T}_{1}$ and $\mathscr{T}_{2}$ are distinct members of $\Sigma$, then $\left(F, \mathscr{T}_{1}\right)$ and $\left(F, \mathscr{T}_{2}\right)$ are not topologically isomorphic. The analogous statement for commutative rings without proper zero-divisors is also true.

Proof. Let $\Sigma^{\prime}$ be the family of all field topologies on $F$. Then, by Corollary $2.2,\left|\Sigma^{\prime}\right|=2^{2^{|p|}}$. Let $\Sigma$ be a set which contains one topology out of each equivalence class on $\Sigma^{\prime}$ for the equivalence relation $\mathscr{T}_{1} \sim \mathscr{T}_{2}$ iff $\left(F, \mathscr{T}_{1}\right)$ is topologically isomorphic to $\left(F, \mathscr{T}_{2}\right)$. Since there are at most $\left|F^{F}\right|=2^{\left|F^{\mid}\right|}$(see $[9$, p. 417]) field automorphisms of $F$, each equivalence class has at most $2^{\left|F^{\prime}\right|}$ elements in it. But then $2^{2^{|P|}}=\left|\Sigma^{\prime}\right| \leqq 2^{\left|F^{\prime}\right|} \cdot|\Sigma|$. The only way this cardinal inequality can be satisfied is if $2^{2} \leqq|\Sigma|$. Clearly then $|\Sigma|=2^{2^{|P|}}$.

3. Suprema of nonlocally bounded topologies. The $2^{2^{|f|}}$ field topologies produced on $F$ in $\$ 2$ are suprema of valuation topologies, which are, of course, locally bounded. We outline here how it can be shown that a field $F$ of infinite transcendence degree over a subfield also admits $2^{2^{|P|}}$ field topologies which are not the suprema of locally bounded ring topologies.

We will use the same notation in this section as in $\S 2$. The basic idea will be to define a nonlocally bounded topology $\mathscr{T}_{S}^{\prime}$ on $F$ for each $S \in \mathscr{S}$ such that, for each $\alpha \in X,\left(\phi_{n}(\alpha)\right)$ converges to zero in $\mathscr{T}_{S}^{\prime}$ if $\alpha \in S$ and is bounded away from zero otherwise. One sees that this is precisely what made the proof of Theorem 2.1 work.

To get these topologies, we modify the inductive procedure of [5] somewhat. This procedure involved inductively building up a basis $V_{0} \supseteq V_{1} \supseteq \cdots$ of neighborhoods of zero for a ring topology $\mathscr{T}\left(\left(a_{n}\right),\left(C_{n}\right)\right)$ on $F$ such that $a_{n} \in V_{n}$ for each $n$ and such that $C_{n+1} \cdot V_{n+1} \subseteq V_{n}$ for each set $C_{n+1}$ in a nested sequence $C_{1} \subseteq C_{2} \subseteq \cdots$ of subsets of $F$. This procedure can easily be generalized to yield a ring topology $\mathscr{T}\left(\left(A_{n}\right),\left(C_{n}\right)\right)$, where now the $A_{n}$ 's are subsets of $F$, and for each $n, A_{n} \subseteq V_{n}$.

In what follows, $K_{0}, K_{1}, \cdots$ will be a sequence of subfields of $F$ defined by $K_{0}=K$, and, for each $n \geqq 0, K_{n+1}$ equals the algebraic closure of $K_{n}\left(B_{n}\right)$ 
in $F$. We note that $K_{n} \subseteq K_{n+1}$, that $\bigcup_{n=0}^{\infty} K_{n}=F$, and that $\bigcup_{i=n}^{\infty} B_{i}$ is algebraically independent over $K_{n}$.

For each $S$ in $\mathscr{S}$, we will take $\mathscr{T}_{S}^{\prime}$ to be the inductive ring topology $\mathscr{T}\left(\left(\phi_{n}(S)\right),\left(K_{n}\right)\right)$. By this definition, it is automatically the case that for $\alpha \in S$, the sequence $\left(\phi_{n}(\alpha)\right)$ converges to zero in $\mathscr{T}_{S}^{\prime}$. The following lemma will assure that $\left(\phi_{n}(\alpha)\right)$ is bounded from zero if $\alpha$ is not in $S$, and will assure that suprema of sets of these topologies are of the desired type.

LEMMA 3.1. Let $\left\{V_{n} \mid n \geqq 0\right\}$ denote the inductively generated basis for the inductive ring topology $\mathscr{T}_{S}^{\prime}$. If $x$ is in $V_{n} \cap\left(K_{m+1} \sim K_{m}\right)$, then $x$ has the form $P\left(a_{1}, \cdots, a_{k}\right)$, where:

(a) $P\left(Y_{1}, \cdots, Y_{k}\right)$ is a polynomial with coefficients in $K_{m}$;

(b) The degree of $P$ in any one of the indeterminates $Y_{i}$ is at most $2^{i n}$; and

(c) The $a_{i}$ 's are all in $\bigcup_{j=1}^{m} \phi_{j}(S)$.

Proof. To define the topology $\mathscr{T}\left(\left(\phi_{n}(S)\right),\left(K_{n}\right)\right)$, one would modify the development in $[5, \S 1]$ merely by putting an indeterminate for each element of $\phi_{k+1}(S)$ into the set $W_{k+1}^{k+1}$ defined in [5, (1.10), p. 151], rather than just putting one indeterminate into this set. One may check that the lemmas in [5, §2], suitably modified, would still hold.

In particular, Lemma 2.2, (1.19), and (1.12) of [5] would assure that $x=P\left(a_{1}, \cdots, a_{k}\right)$, where, for some integer $r$, (i) $\left\{a_{i} \mid 1 \leqq i \leqq k\right\} \subseteq \bigcup_{j=1}^{r} \phi_{j}(S)$; (ii) $P\left(Y_{1}, \cdots, Y_{k}\right)$ has coefficients in $K_{r}$; (iii) the degree of $P$ in any $Y_{i}$ is at most $2^{r}$; (iv) at least one $a_{i}$, say $a_{k}$, is in $\phi_{r}(S)$; and (v) the degree of $P\left(a_{1}, \cdots, a_{k-1}, Y_{k}\right)$ in $Y_{k}$ is at least one. (Some of the details of the proof parallel those of $[5, \S 4]$.) Thus, $\left\{a_{i} \mid 1 \leqq i \leqq k\right\} \subseteq \bigcup_{j=1}^{r} B_{j}$, while $a_{k}$ and perhaps others of the $a_{i}$ 's are in $B_{r}$. It follows that $x \in K_{r+1}$. Since $a_{k} \in B_{r}$, and $B_{r}$ is algebraically independent over $K_{r}$, it follows that $x \notin K_{r}$, for if it were then, by $(v), x=P\left(a_{1}, \cdots, a_{k}\right)$ would be an equation of algebraic dependence over $K_{r}$ for elements in $B_{r}$, which is a contradiction. Thus, $x \in K_{r+1} \sim K_{r}$, so it follows that $r=m$. Statements (a)-(c) now follow from (i)-(iii).

As a consequence of Lemma 3.1, one has that if $\alpha \in X \sim S$, then $\phi_{n}(\alpha) \notin V_{0}$ for each $n$. Since $\phi_{n}(\alpha) \in B_{n}, \phi_{n}(\alpha) \in K_{n+1} \sim K_{n}$. Thus, if $\phi_{n}(\alpha) \in V_{0}$, then $\phi_{n}(\alpha)=P\left(a_{1}, \cdots, a_{k}\right)$, with $P$ and the $a_{i}$ 's as specified in Lemma 3.1. Since $\phi_{n}(\alpha) \notin \bigcup_{j=1}^{n} \phi_{j}(S)$, (a) and (c) show that $\phi_{n}(\alpha)=$ $P\left(a_{1}, \cdots, a_{k}\right)$ is a nontrivial equation of algebraic dependence for $\phi_{n}(\alpha)$, and perhaps other elements of $B_{n}$, over $K_{n}$, which is a contradiction.

THEOREM 3.2. For distinct, nonempty subsets $\mathcal{O}$ of $\mathscr{S}$, the ring topologies $\mathscr{T}_{\mathcal{O}}^{\prime}=\sup \left\{\mathscr{T}_{S}^{\prime} \mid S \in \mathcal{O}\right\}$ are distinct, and fail to be suprema of locally bounded ring topologies on $F$. Thus, a field $F$ of infinite transcendence degree over $a$ subfield admits $2^{2^{|F|}}$ ring topologies of this latter type. 
Proof. As we have noted, the fact that $\left(\phi_{n}(\alpha)\right)$ converges to zero in $\mathscr{T}_{S}^{\prime}$ if $\alpha \in S$ and is bounded from zero if $\alpha \notin S$ assures that the proof of Theorem 2.1 will adapt to prove the first assertion.

To prove the second assertion, we use a result of J. Heine [3] which says that $\mathscr{T}_{\mathscr{O}}^{\prime}$ is not the supremum of a family of locally bounded ring topologies on $F$ provided that there is a $\mathscr{T}_{\mathcal{U}}^{\prime}$-neighborhood $U$ of zero such that for every $\mathscr{T}_{\mathcal{C}}^{\prime}$-neighborhood $V$ of zero, there is an integer $m$ such that $V^{m} \notin U$.

To get such a neighborhood $U$, we fix any $S \in \mathcal{O}$, and let $U=V_{0}$, the 0 th neighborhood of zero in the inductively defined basis for $\mathscr{T}_{S}^{\prime}$. Then $U$ is also a $\mathscr{T}_{\mathcal{O}}^{\prime}$-neighborhood of zero. If $V \in \mathscr{T}_{\mathscr{O}}^{\prime}$ and $0 \in V$, then there are sets $S_{1}, S_{2}, \cdots, S_{k}$ in $\mathcal{O}$, and for each $i$ a $\mathscr{T}_{S_{i}}^{\prime}$-neighborhood $W_{i}$ of zero such that $\bigcap_{i=1}^{k} W_{i} \subseteq V$. Since $\mathscr{S}$ has the f.i.p.c., there is an element $\alpha$ in $\bigcap_{i=1}^{k} S_{i}$. Then the sequence $\left(\phi_{n}(\alpha)\right)$ converges to zero in each of the topologies $\mathscr{T}_{S_{i}}^{\prime}$, so for a sufficiently large integer $m, \phi_{m}(\alpha) \in \bigcap_{i=1}^{k} W_{i}$.

We claim that $\phi_{m}(\alpha)^{r} \notin U=V_{0}$, where $r=2^{m}+1$. Suppose otherwise. Then since $\phi_{m}(\alpha) \in B_{m}, \phi_{m}(\alpha)^{r} \in V_{0} \cap\left(K_{m+1} \sim K_{m}\right)$, so by Lemma 3.1, $\phi_{m}(\alpha)^{r}=P\left(a_{1}, \cdots, a_{k}\right)$, where $P$ has coefficients in $K_{m}$, the $a_{i}$ 's are in $K_{m}$ or in $B_{m}$, and the degree of $P$ in each indeterminate is at most $2^{m}$. This equation then gives a nontrivial equation of algebraic dependence for $\phi_{m}(\alpha)$, and perhaps other elements of $B_{m}$, over $K_{m}$, a contradiction.

We thus have that $V^{r} \ddagger U$, and the desired result is proven.

REMARK. We wish to note here that Theorem 3.2 can be strengthened to prove the existence of $2^{2^{|x|}}$ field topologies on $F$ which are not suprema of locally bounded ring topologies. To do so, one needs to make more extensive modifications in the methods of [5]. Specifically, in order to build continuity for multiplicative inversion into an inductive topology, one must work with rational functions rather than polynomials, (such as appeared in Lemma 3.1). However, a presentation here of all the technical details is perhaps not justified for the sake of this relatively minor improvement in the result.

We conclude with two further questions of the type considered herein which to the writer's knowledge remain unanswered.

1. In the proof of Theorem 2.1, we displayed $2^{\left|F^{*}\right|}$ first countable, locally bounded field topologies (the maximum number possible) on a field $F$ of infinite transcendence degree over a subfield. The only fields not of this type are those of finite transcendence degree over their prime subfields. How many first countable, locally bounded field topologies are there on a field of this latter type? It is known [5, Theorem 6.1, p. 159] that if $F$ is algebraic over its finite prime subfield, then there are two, namely the discrete and indiscrete topologies. Otherwise, there are at least countably many determined by valuations. In particular, it would be of 
interest to know how many there are on the rational numbers and on a simple transcendental extension of a field of prime order.

2. We have seen that on a field $F$ of infinite transcendence degree, there are as many first countable, locally bounded field topologies as possible, namely $2^{|F|}$. Are there as many nonfirst countable, locally bounded ones as possible, namely $2^{2^{|F|}}$ ?

ADDED IN PROOF. Results close to those obtained herein have just recently been published by J. Heine [Ringtopologien auf nichtalgebraischen Körpern, Math. Ann. 199 (1972), 205-211]. By quite different means, he obtains, for any nonalgebraic field $F, 2^{2^{|P|}}$ ring topologies which are, and $2^{2^{|P|}}$ ring topologies which are not suprema of locally bounded ones. Thus, this paper extends these results for most nonalgebraic fields by obtaining like numbers and types of field topologies. It does not appear that Heine's methods generalize to give field topologies. However, Podewski writes that he now has extended his methods to the uncountable case. Heine also points out in correspondence that question 2 above has an easy negative answer; since a locally bounded topology on $F$ is uniquely determined by a bounded neighborhood of zero, there can be at most $2^{|F|}$ of them, whether or not they are first countable.

\section{REFERENCES}

1. N. Bourbaki, Éléments de mathématique. Livre III: Topologie générale. Chaps. 3-4, Actualités Sci. Indust., no. 1143, 3rd. ed., Hermann, Paris, 1960; English transl., Hermann, Paris; Addison-Wesley, Reading, Mass., 1966. MR 25 \#4021; MR 34 \#5044a.

2. F. Hausdorff, Über zwei Sätze von G. Fichtenholz und L. Kantorovich, Studia Math. 6 (1936), 18-19.

3. J. Heine, Existence of locally unbounded topological fields, and field topologies which are not the intersection of bounded ring topologies, J. London Math. Soc. (2) 5 (1972), 481-487.

4. L. Hinrichs, Integer topologies, Proc. Amer. Math. Soc. 15 (1964), 991-995. MR 31 \#2286.

5. J. Kiltinen, Inductive ring topologies, Trans. Amer. Math. Soc. 134 (1968), 149-169. MR 37 \#4054.

6. - Inverses of polynomial functions in topological fields, Proc. Amer. Math. Soc. 24 (1970), 9-17. MR 40 \#4250.

7. K.-P. Podewski, The number of field topologies on countable fields, Proc. Amer. Math. Soc. 39 (1973), 33-38.

8. O. Schilling, The theory of valuations, Math. Surveys, no. 4, Amer. Math. Soc., Providence, R.I., 1950. MR 13, 315.

9. W. Sierpiński, Cardinal and ordinal numbers, 2nd rev. ed., Monografie Mat., vol. 34, PWN, Warsaw, 1965. MR 33 \#2549.

Department of Mathematics, Northern Michigan University, Marquette, Michigan 49855 